\title{
REVIEW
}

\section{A Review of the Efficacy of Thalidomide and Lenalidomide in the Treatment of Refractory Prurigo Nodularis}

\author{
Victoria M. Lim • Eric L. Maranda · Vivek Patel · Brian J. Simmons • \\ Joaquin J. Jimenez
}

Received: April 29, 2016/ Published online: June 11, 2016

(c) The Author(s) 2016. This article is published with open access at Springerlink.com

\section{ABSTRACT}

Prurigo nodularis (PN) is a chronic dermatoses characterized by intensely pruritic, excoriated, or lichenified nodules. Standard therapy includes corticosteroids, antihistamines, and phototherapy; however, treatment results are often inadequate or transient. Thalidomide and its analogue lenalidomide are immunomodulatory drugs that have successfully been used to treat refractory cases of PN. A systematic review was performed evaluating the use of thalidomide and lenalidomide for PN. Eighteen articles were

Enhanced content To view enhanced content for this article go to http://www.medengine.com/Redeem/ F6D4F0602F038A59.

Victoria M. Lim and Eric L. Maranda are co-first authors and equally contributed.

V. M. Lim

Creighton University School of Medicine, Omaha,

NE 68102, USA

E. L. Maranda $(\bowtie)$. V. Patel · B. J. Simmons .

J. J. Jimenez

Department of Dermatology and Cutaneous

Surgery, University of Miami Miller School of

Medicine, 1475 NW 12th Ave., Suite 2175,

Miami, FL 33136, USA

e-mail: emaranda@med.miami.edu included in this study in which a total of 106 patients were evaluated, of whom 76 (71.7\%) had moderate to significant improvement of PN with the use of thalidomide, lenalidomide, or both. Patients given thalidomide were treated with doses of 50-300 mg daily for 1-142 months, with the majority being treated for less than 1 year. Patients treated with lenalidomide were given a daily dose of $5-10 \mathrm{mg}$ from 3 to 24 months. The most common side effects observed were sedation, gastrointestinal symptoms, and transient peripheral neuropathy. While thalidomide and lenalidomide are drugs that have shown promising results in these studies, caution should be taken in prescribing these medications and patients should be informed about the potential side effects. As such, large-scale randomized controlled trials with long-term follow-up are needed to determine appropriate dosing, efficacy, and toxicity profiles.

Keywords: Nodular prurigo; Lenalidomide; Prurigo nodularis; Pruritus; Thalidomide; Treatment 


\section{INTRODUCTION}

Prurigo nodularis (PN) of Hyde, also known as nodular prurigo, is a chronic skin disease of unknown etiology. It is characterized by an intensely pruritic papulonodular eruptions. The hyperkeratotic lesions tend to be symmetrical and occur over the extensor surfaces of the extremities. $\mathrm{PN}$ has been associated with a range of systemic conditions such as HIV/AIDS, depression, gastrointestinal disorders, hypothyroidism, and hematologic malignancies. In most cases, however, the etiology is unclear [1-4]. First-line therapy for $\mathrm{PN}$ is comprised of topical antipruritics or corticosteroids, oral steroids, antihistamines, or certain antidepressants such as doxepin [5]. Additional commonly used treatments include phototherapy, capsaicin, cyclosporine, and methotrexate [3]. Recently, novel agents such as tumor necrosis factor (TNF) inhibitors alfacept and etanercept have also been prescribed [14]. Most of these therapies, however, are ineffective or only provide temporary relief. Thus, alternative treatments are needed for refractory cases.

In 1965, Sheskin was the first to report on the use of thalidomide to treat a patient with PN [6]. Thalidomide is a drug with numerous proposed mechanisms of action and effects, ranging from acting as a prostaglandin/ histamine antagonist to being an inhibitor of fibroblast growth factor and a immunomodulatory agent [7]. As an immunomodulatory drug, thalidomide functions to decrease TNF- $\alpha$ and inhibits polymorphonuclear leukocyte chemotaxis [7]. TNF- $\alpha$ induces pain by increasing the production of interleukin (IL)-1b and IL-6, which then increases the production of IL-8, a known mediator of sympathetic pain [8-10]. More recently, thalidomide has also been shown to inhibit the peripheral vanilloid receptor 1 (TRVP1) channel, which is also involved in pain sensation [11].

Thalidomide became well known for its teratogenic effects leading to severe fetal malformations when taken by pregnant women before the third trimester [12]. This medication has other known side effects, which include peripheral neuropathy, somnolence, altered mood, thrombosis, and gastrointestinal symptoms.

Lenalidomide, a more potent analogue of thalidomide, has been most commonly used to treat multiple myeloma and myelodysplastic syndromes. Lenalidomide's molecular structure varies by one less carbonyl group but one additional amine compared to thalidomide [13]. This change confers a different toxicity profile and, as such, lenalidomide has a much lower frequency of peripheral neuropathy [14]. While some studies report the use of thalidomide and lenalidomide to be beneficial for the treatment of $\mathrm{PN}$, their use in the management of $\mathrm{PN}$ remains controversial. Thus, this review aims to explore and review the efficacy and safety of thalidomide and lenalidomide for the treatment of refractory $\mathrm{PN}$.

\section{METHODS}

A systematic literature review was performed to identify articles between January 1, 1970 and February 15, 2016 relevant to the treatment of prurigo nodularis with thalidomide or lenalidomide. The terms "prurigo nodularis OR nodular prurigo OR picker's nodules OR lichen corneus obtusus OR atypical nodular form of neurodermatitis circumscripta" were searched in the National Library of Medicine's PubMed Database and returned 350 articles. Exclusion criteria included articles that were not written in English and those that were not original 


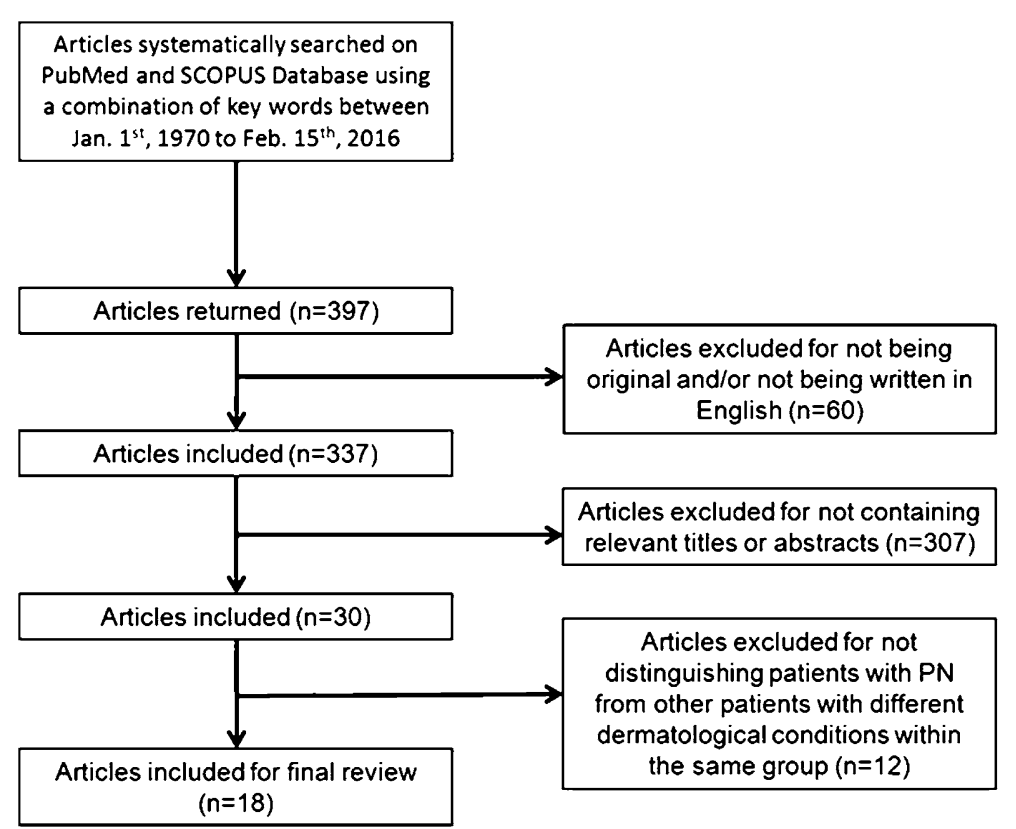

Fig. 1 Flowchart of search methodology. $P N$ prurigo nodularis

clinical trials, case series or case reports. Thirty manuscripts with relevant titles and abstracts were included for further review, based on case reports/series due to lack of controlled studies. If a study with a cohort or group did not distinguish patients with PN from other patients with different dermatological conditions within the same group, it was excluded. In addition, the SCOPUS Database was crosschecked, but no additional articles were found. As a result, a total of 18 articles were selected for inclusion in this review (Fig. 1). This article is based on previously conducted studies and does not involve any new studies of human or animal subjects performed by any of the authors.

\section{RESULTS}

In the first reported case, Van de Broek et al. described a 57-year-old man with refractory PN admitted for an infection superimposed on nodules that had previously failed both topical and oral steroids as well as antihistamines [12]. Thalidomide $100 \mathrm{mg}$ bis in die (BID) was used for 3 months and discontinued after resolution of the pruritis and lesions. No side effects were reported.

Andersen and Fogh retrospectively reviewed 42 subjects with PN treated with thalidomide [22]. Of these, $60 \%$ of patients showed clinical improvement, but $9.5 \%$ of patients showed no effect. The average daily dose was $100 \mathrm{mg}$, though a few patients received $50 \mathrm{mg}$ due to gastrointestinal-related side effects. The mean duration of treatment was 105 weeks, and $59.5 \%$ of patients developed peripheral neuropathy ranging from 1 week to 7.5 years after initiation of treatment, prompting discontinuation of therapy at the time of symptom development. None of the subjects with neuropathy showed evidence of this adverse effect during follow-up.

Alfadley et al. started thalidomide $100 \mathrm{mg}$ BID in a Hepatitis B patient who developed major depressive disorder and suicidal risk 
following a 5-year history of PN refractory to therapy [17]. After 3 weeks of treatment, the pruritus had mildly decreased without improvement in skin lesions. Subsequently, the dose was increased to $300 \mathrm{mg}$ daily. By the 8th month, the pruritus had ceased, the majority of lesions had cleared, and mild sensory loss developed. The patient was then placed on a maintenance dose of $50 \mathrm{mg}$ every other day for 3 months. At the 4-month follow-up, the patient remained disease-free and the sensory loss had dramatically improved.

Maurer et al. performed a trial on 10 HIV-infected patients. They received thalidomide $100 \mathrm{mg}$ per day and were randomized after 1 month to receive either 100 or $200 \mathrm{mg}$ daily [4]. Doses were adjusted if adverse events appeared, thus daily dosing ranged from 33 to $200 \mathrm{mg}$. Eight patients (80\%) continued the trial longer than 1 month, and all had a greater than 50\% reduction in itch, while 7 (70\%) had a greater than 50\% reduction in lesions. Pruritic reduction occurred within 1-3 months of treatment, while decreases in erythema and nodule size occurred between 3-6 months. Three patients (30\%) developed peripheral neuropathy; however, no association with neuropathy was found between treatment duration, daily dose, or cumulative dose.

Herranz et al. also treated a case of AIDS-associated $\mathrm{PN}$ in a 35-year-old white male with a CD4 count of 8 cells $/ \mathrm{mm}^{3}$ [2]. Following a failed trial of topical corticosteroids and antihistamines, he was placed on thalidomide $100 \mathrm{mg}$ daily for 6 weeks, upon which there was a drastic improvement of itching and lesions. The patient had a relapse of tuberculosis after 1 month of discontinuing thalidomide, which was postulated to have been due to the inhibitory effect of thalidomide on TNF-a. There were no other side effects reported.

Ferrandiz et al. conducted a prospective trial to evaluate combination therapy using thalidomide and ultraviolet-B (UVB) irradiation on four patients with PN [15]. Two patients had previously undergone a course of thalidomide but subsequently relapsed. Thalidomide was started at $100 \mathrm{mg}$ daily and was discontinued once significant clinical improvement was achieved, ranging from 8 to 16 weeks. UVB phototherapy was then initiated for three sessions per week until complete clearance of the nodules was achieved, with a maximum of 70 exposures allowed. Most symptoms resolved after 6-12 UVB treatments, at which point thalidomide was also discontinued. One patient developed temporary peripheral neuropathy and another developed nausea. The follow-up period ranged from 4-18 months, in which one patient experienced a relapse at 5 months, which was then successfully managed with 35 treatments of UVB irradiation.

Lenalidomide has also been used in patients experiencing toxicity with thalidomide. Kanavy et al. treated a 45-year-old black woman with a severe 10-year case of PN [14]. She was first treated with thalidomide $200 \mathrm{mg}$ daily and had a dramatic improvement, but she developed neuropathy after 18 months and treatment was stopped. After thalidomide, she also was treated with oral naltrexone, minocycline, gabapentin, alafecept, and etanercept with no sucess [14]. Thereafter, she was started on lenalidomide $5 \mathrm{mg}$ a day and by the second week she noted a significant decrease in pruritus, sleep, and concentration ability. By the first month, she exhibited healing of nodules and disappearance of itching. Two attempts were made to taper her dose and both resulted in disease flare-ups, thus she was maintained on $5 \mathrm{mg} /$ day for a total of 
24 months. The patient remained in remission after 2 years. Liu et al. reported another case of lenalidomide-treated refractory PN. The patient had a severe 20-year history of the disease. She was previously treated with steroids, antihistamines, and underwent psoralen + UVA therapy for 6 months with no relief. Furthermore, the latter treatment resulted in the development of squamous cell carcinomas on her lower extremities. After discontinuation of the photochemotherapy, she was started on $100 \mathrm{mg}$ of thalidomide daily. By month 3, she developed tingling and numbness in all of her limb extremities and thalidomide was discontinued. After 1 month, she resumed thalidomide at $50 \mathrm{mg}$ but her neuropathy returned and the medication was discontinued. Four months later, she was started orally on lenalidomide $10 \mathrm{mg} /$ day with a marked reduction in nodules and itching intensity after only 1 month. Her reported adverse effects were arthralgias, nausea, and vomiting. She continued taking lenalidomide and achieved almost complete clearance until right leg weakness prompted termination of the current treatment after 3 months of lenalidomide. She had no residual neuropathy, but her PN relapsed.

\section{TRENDS IN TREATMENT, DOSAGE, AND LENGTH}

A total of 18 studies with 106 patients treated with thalidomide or lenalidomide for PN were reviewed (see Tables 1, 2). It should be noted that these trends were based on case reports/ series or retrospective literature due to lack of controlled studies, especially in regards to lenalidomide treatment, as only a few case reports exist. In the earlier studies, patients were started on higher doses of thalidomide of
$200 \mathrm{mg}$ or more daily $[5,12]$. In the majority of studies since then, however, patients were started on an initial dose ranging from 50 to $200 \mathrm{mg}$ thalidomide per day and then tailored according to response or development of side effects. Length of treatment for thalidomide ranged from 1 month to almost 12 years depending on remission of disease or discontinuation due to development of toxicity. In the study by Ferrandiz et al., patients were treated with combination therapy of thrice weekly narrow-band UVB phototherapy and thalidomide at $100 \mathrm{mg}$ daily for 2.5-5 months [15]. Excellent responses were achieved after an average of 3 months on thalidomide and 32 UVB courses [15]. One patient that was taking lenalidomide was kept on $5 \mathrm{mg}$ for over 2 years, while the other took $10 \mathrm{mg}$ daily for 3 months [11, 13]. For lenalidomide, treatment length was from 3 to over 24 months. Lenalidomide offered a relatively low toxicity treatment to patients who had toxicity to thalidomide.

\section{TRENDS IN OUTCOMES}

A total of 106 patients were included in this review, 76 (71.7\%) of whom showed significant improvement in reduction of pruritus and disappearance of nodules [1, 2, 4, 14-28]. Only slight or mild improvement was seen in $11(10.3 \%)$ patients $[23,25,26]$, while 15 (13.3\%) patients showed no improvement at all $[19,23,25,26,29]$. Results were unable to be determined in four patients in one retrospective study [25]. Pruritus typically was the first to decrease with a noticeable clinical difference by $2-4$ weeks followed by flattening of lesions and decreased excoriation which occurred at an average of 2 months. For patients who achieved complete or almost 


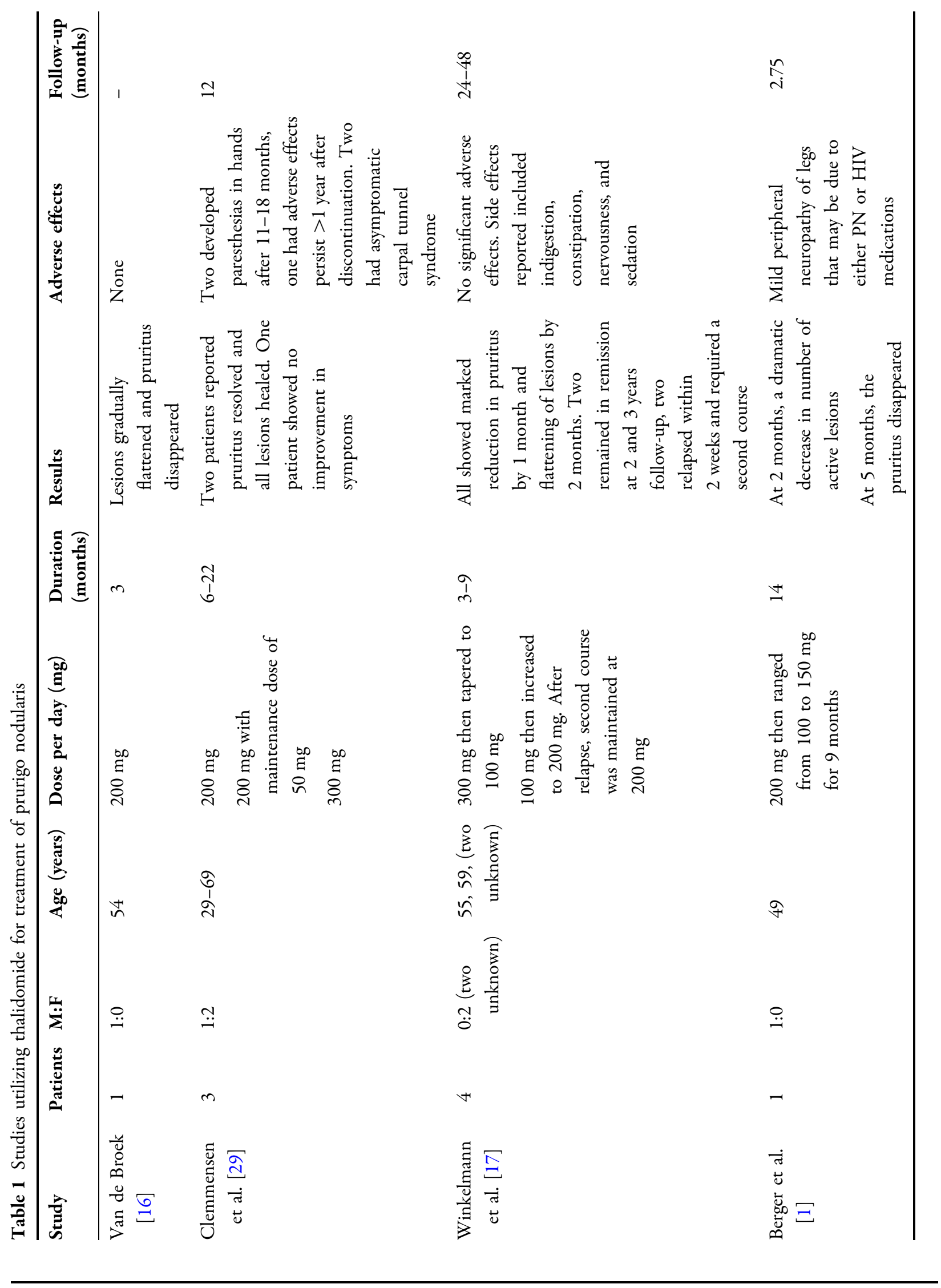


Dermatol Ther (Heidelb) (2016) 6:397-411

403

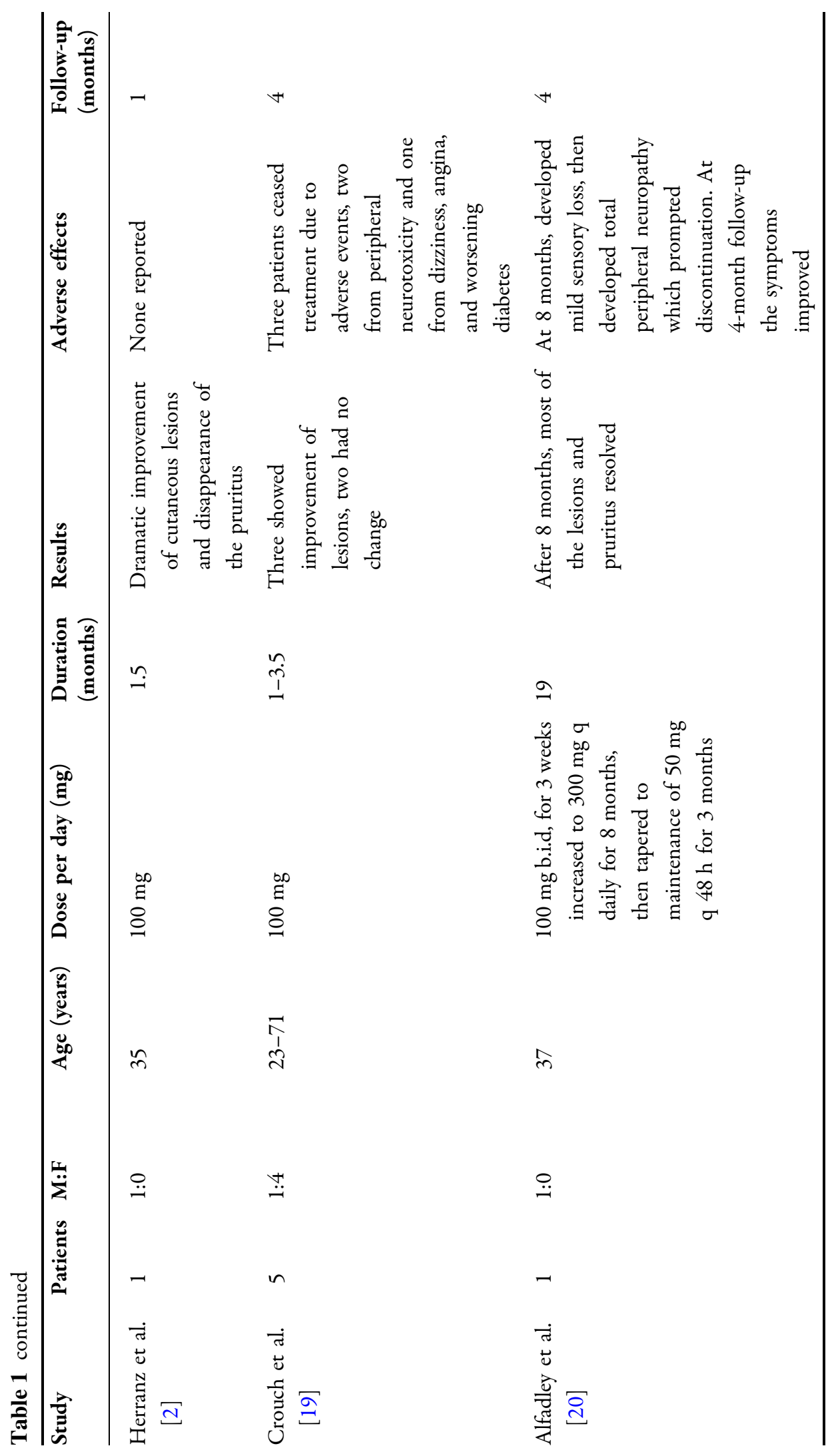

$\triangle$ Adis 


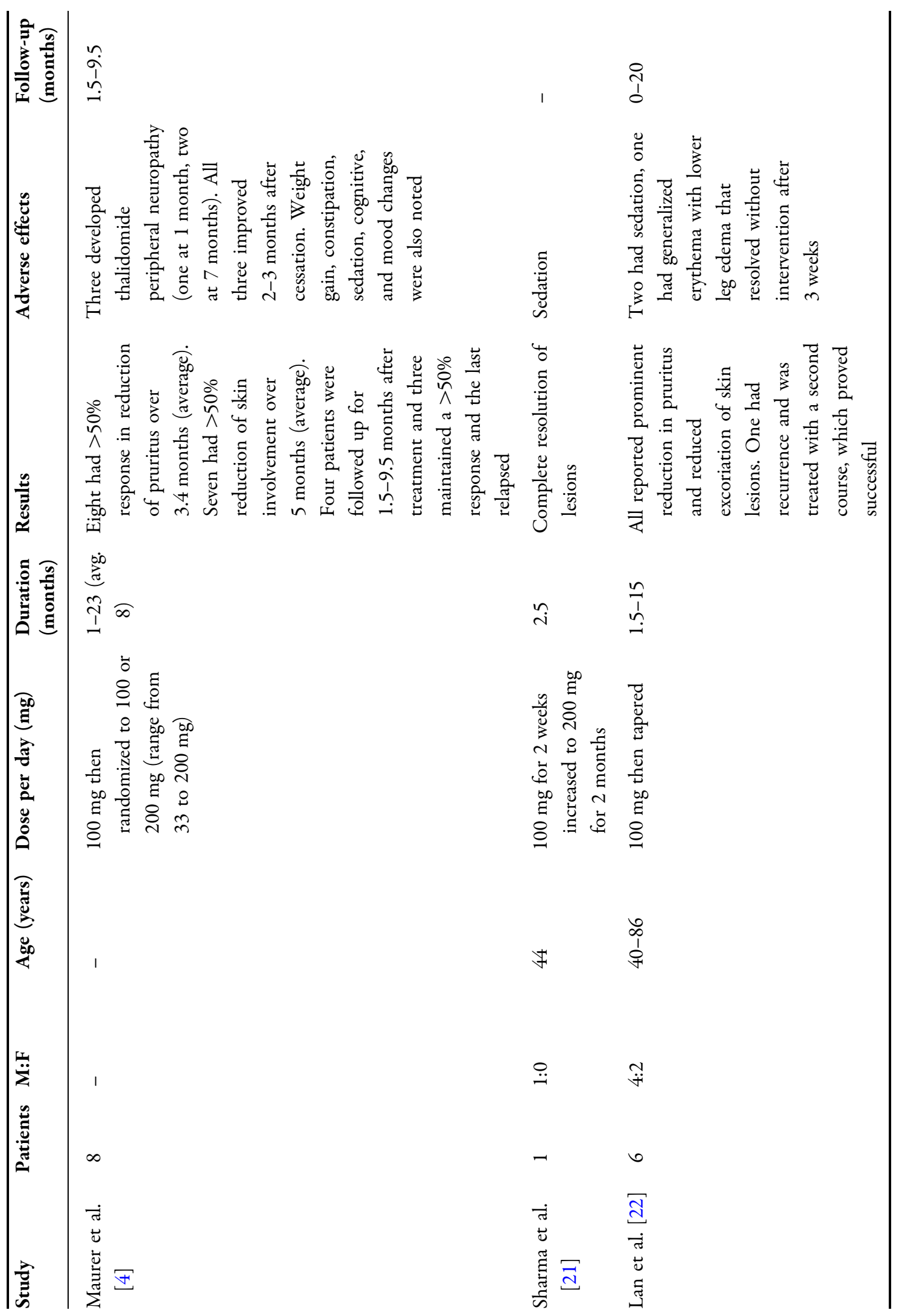




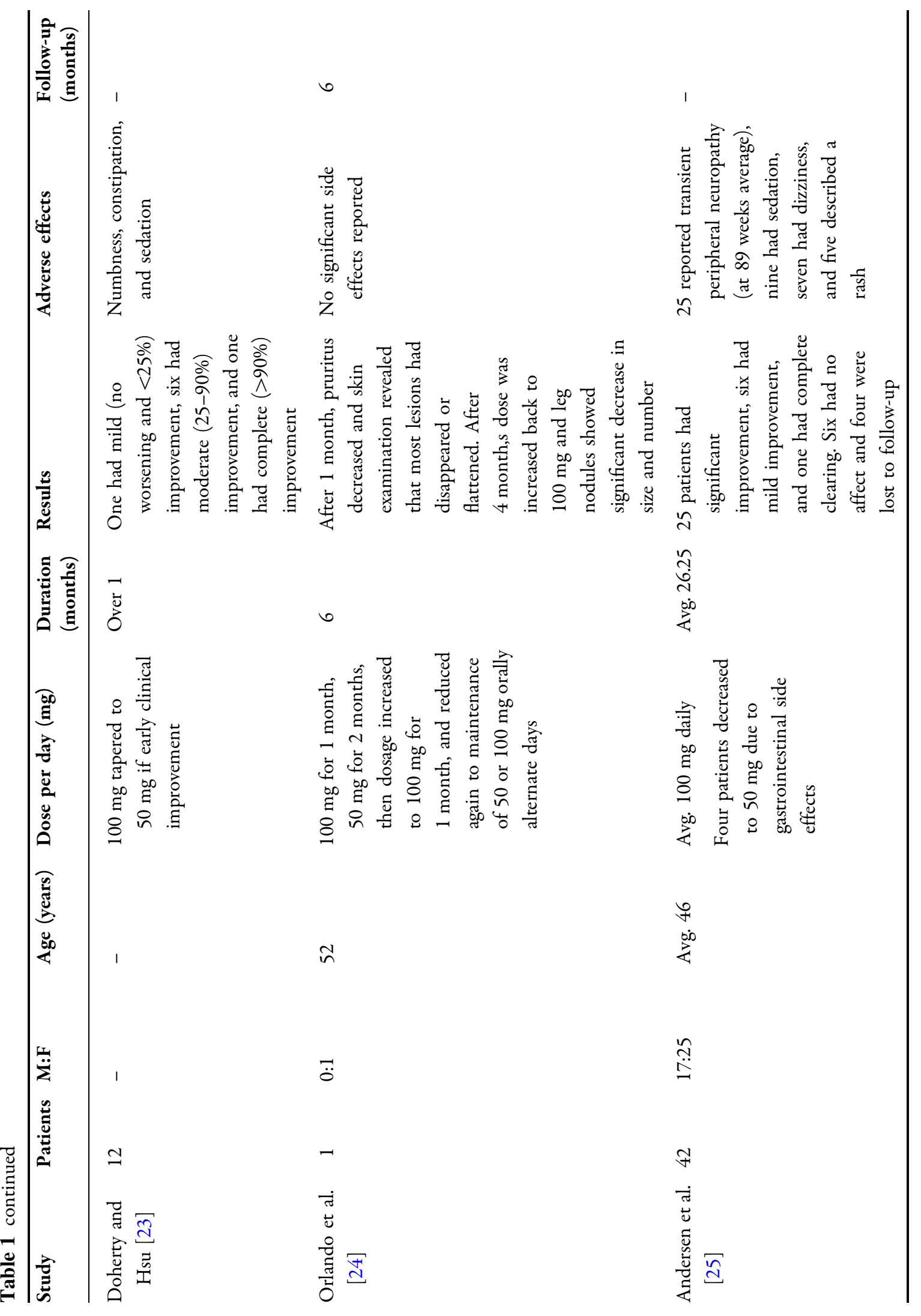




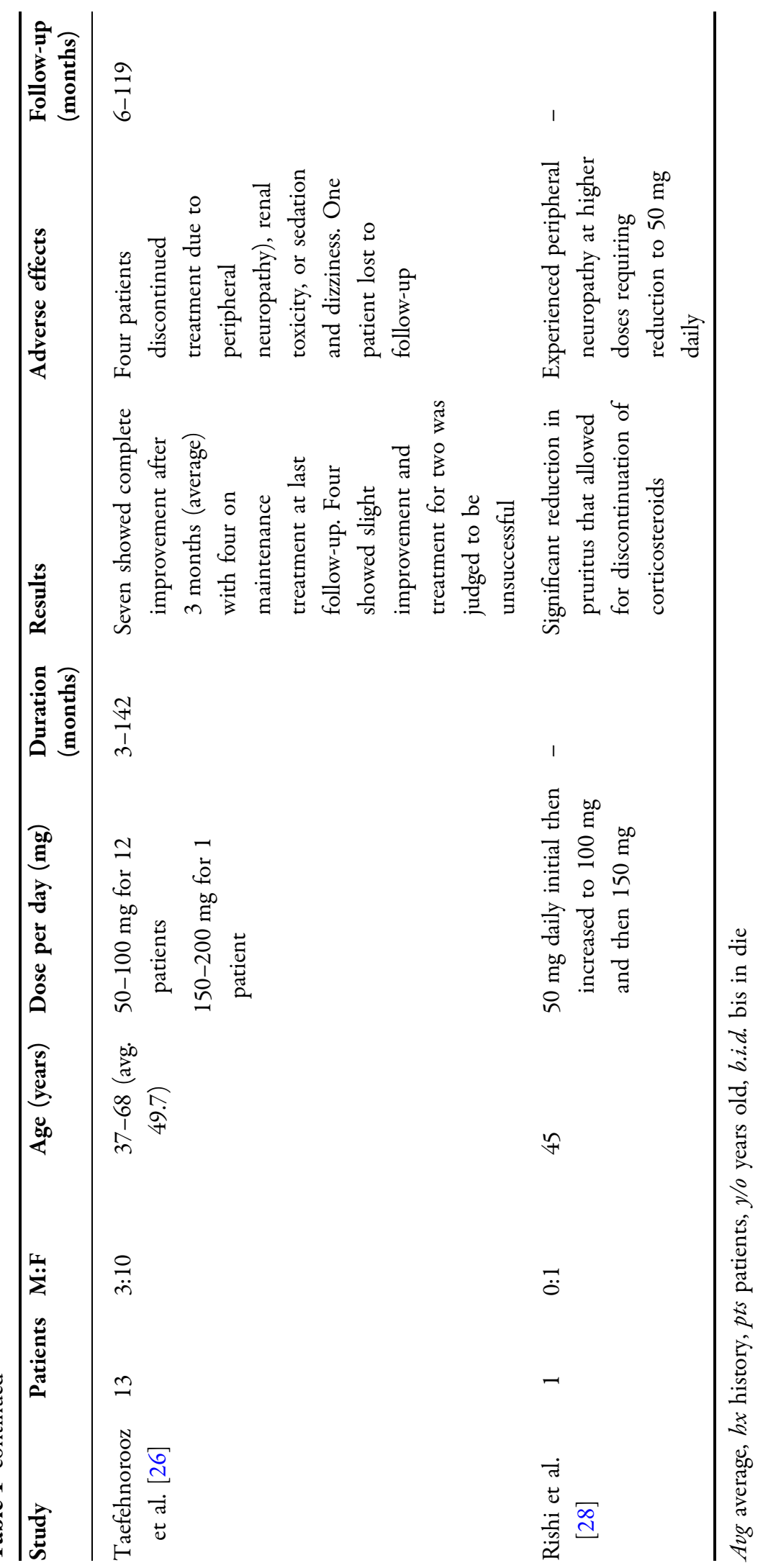




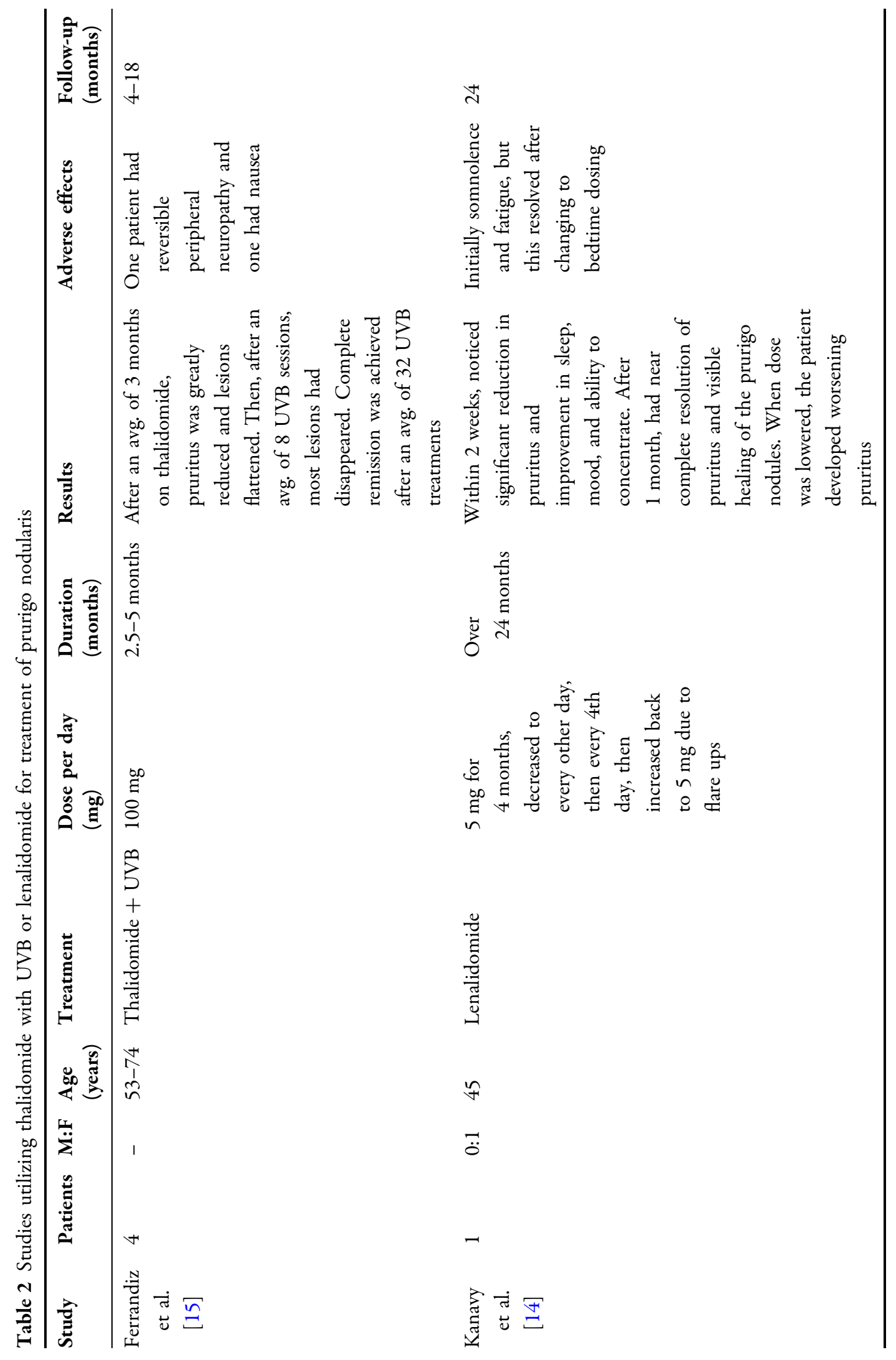




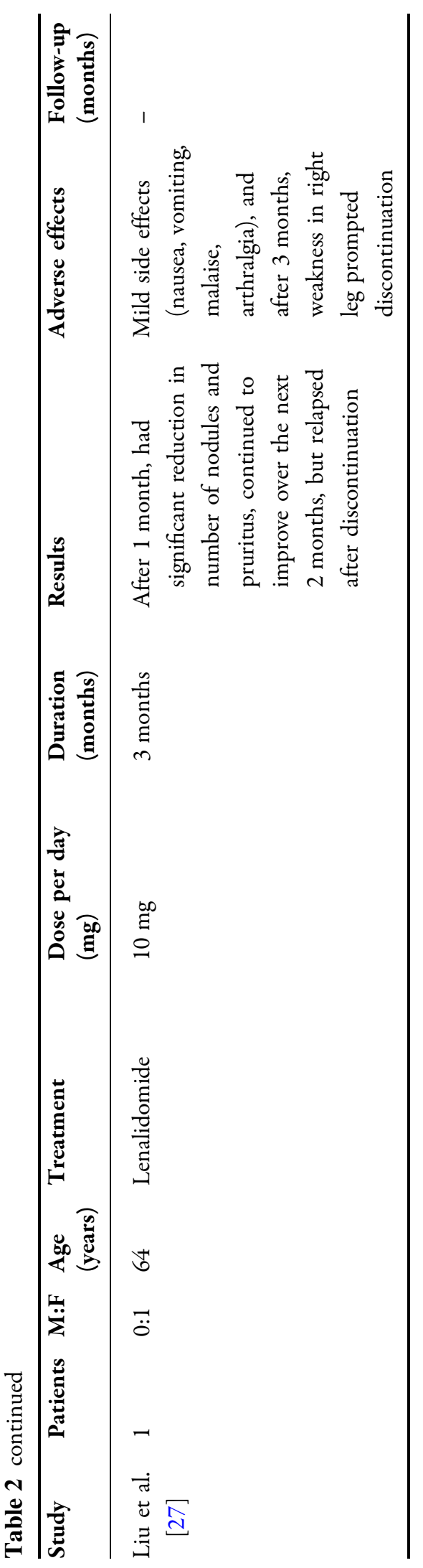

complete remission, time until that endpoint varied from as little as 1 month to as long as 8 months [20].

\section{TRENDS IN ADVERSE EFFECTS}

Peripheral neuropathy was the most common adverse effect. Overall, there were a reported 39 $(36.8 \%)$ cases of peripheral neuropathy $[1,4,15,16,18-20,25-28]$ not including the additional patients affected in Doherty and Hsu's study, as the exact amount was unable to be obtained [23]. Thalidomide-induced neuropathy developed after 1 week to 7.5 years of treatment [25]. In most cases, the peripheral neuropathy was reversible and took up several months to resolve. Some patients with HIV/ AIDS experienced peripheral neuropathy, but it was unclear if it was due to thalidomide or the HIV infection [1]. Clemmensen et al. had one patient who still experienced neuropathy over a year after discontinuation; however, it should be noted that the patient used the drug for 18 months [29]. Although no studies directly or extensively addressed the subject, there appears to be increased toxicity at higher doses of thalidomide $[14,20,22,26]$. Sedation, gastrointestinal symptoms, and dizziness were the other most-often reported adverse effects.

Lenalidomide was used in two patients after they failed thalidomide therapy $[14,27]$. The first patient discontinued thalidomide after developing peripheral neuropathy. She was then treated successfully with lenalidomide and had no recurrence of paresthesias [14]. The other patient also developed tingling and decreased sensation with thalidomide; however, she eventually re-developed neuropathy after a trial of lenalidomide [27]. More studies are needed to determine the outcomes of thalidomide compared to lenalidomide, and 
whether this could be a preferred treatment option with less side effects.

\section{TRENDS IN RECURRENCE}

Seven patients relapsed after thalidomide-induced improvement of PN symptoms and cessation of therapy [14, 15, 17, 22, 27]. Four achieved remission after a second course of completed therapy $[15,17,22]$, and one continued to have disease flare-ups with multiple attempts at tapering the dose, and thus was maintained on $5 \mathrm{mg}$ /day of lenalidomide [14]. The last one had to stop treatment after courses of both thalidomide and lenalidomide due to neuropathy, and relapsed without further resolution [27].

\section{CONCLUSION}

Prurigo nodularis is a severely pruritic skin disease and standard therapy often yields unsatisfactory results. Thalidomide and lenalidomide can be effective alternative treatments for refractory $\mathrm{PN}$, though optimal dosage and treatment time varied in the reviewed studies. Clinical data compiled in this review show that individuals with severe disease experienced the most significant reduction in pruritus and improvement in the appearance of skin. Historically, thalidomide has been used with reluctance due to toxicity; however, the incidence and persistence of adverse effects including neuropathy is highly variable among patients, in the majority of whom clinical improvement preceded the development of permanent deficit. Ideal treatment length and dosing regimens may help abate most side effects. To further avoid adverse effects, thalidomide could be used in sequential combination therapy with UVB or followed by its analogue lenalidomide. Both options shorten the treatment length for thalidomide and may limit adverse reactions; however, most studies are limited by virtue of being case reports and their low power. In the contemporary era of targeted therapy, an enhanced understanding of the biology and related physiology of $\mathrm{PN}$ will allow clinicians to more optimally treat this evasive disease. Additionally, understanding of the molecular mechanisms of thalidomide and lenalidomide may also further elucidate their role in the treatment of PN. Given the findings in the aforementioned studies, thalidomide and its analogues should be further evaluated in a prospective manner with the aim of improving efficacy and reducing treatment-related toxicity by determining the most effective dosing and treatment time.

\section{ACKNOWLEDGMENTS}

No funding or sponsorship was received for this study or publication of this article. All named authors meet the International Committee of Medical Journal Editors (ICMJE) criteria for authorship for this manuscript, take responsibility for the integrity of the work as a whole, and have given final approval for the version to be published.

Disclosures. Victoria M. Lim, Eric L. Maranda, Vivek Patel, Brian J. Simmons and Joaquin J. Jimenez have nothing to disclose.

Compliance with Ethics Guidelines. This article is based on previously conducted studies and does not involve any new studies of human or animal subjects performed by any of the authors. 
Open Access. This article is distributed under the terms of the Creative Commons Attribution-NonCommercial 4.0 International License (http://creativecommons.org/licenses/ by-nc/4.0/), which permits any noncommercial use, distribution, and reproduction in any medium, provided you give appropriate credit to the original author(s) and the source, provide a link to the Creative Commons license, and indicate if changes were made.

\section{REFERENCES}

1. Berger TG, Hoffman C, Thieberg MD. Prurigo nodularis and photosensitivity in AIDS: treatment with thalidomide. J Am Acad Dermatol. 1995;33(5 Pt 1):837-8. doi:10.1016/0190-9622(95)91846-9.

2. Herranz P, Pizarro A, de Lucas R, et al. Treatment of AIDS-associated prurigo nodularis with thalidomide. Clin Exp Dermatol. 1998;23(5):233-4.

3. Lee MR, Shumack S. Prurigo nodularis: a review. Australas J Dermatol. 2005;46(4):211-20. doi:10. 1111/j.1440-0960.2005.00187.x.

4. Maurer T, Poncelet A, Berger T. Thalidomide treatment for prurigo nodularis in human immunodeficiency virus-infected subjects: efficacy and risk of neuropathy. Arch Dermatol. 2004;140(7):845-9. doi:10.1001/archderm.140.7. 845.

5. Saco M, Cohen G. Prurigo nodularis: picking the right treatment. J Fam Pract. 2015;64(4):221-6.

6. Sheskin J. Thalidomide in the treatment of lepra reactions. Clin Pharmacol Ther. 1965;6:303-6.

7. Wu JJ, Huang DB, Pang KR, Hsu S, Tyring SK. Thalidomide: dermatological indications, mechanismis of action and side effects. $\mathrm{Br} \mathrm{J}$ Dermatol. 2005;153:254-73.

8. Marriott JB. TNF-alpha antagonists: monoclonal antibodies, soluble receptors, thalidomide and other novel approaches. Expert Opin Investig Drugs. 1997;6(8):1105-8. doi:10.1517/13543784.6. 8.1105 .

9. McHugh SM, Rowland TL. Thalidomide and derivatives: immunological investigations of tumour necrosis factor-alpha (TNF-alpha) inhibition suggest drugs capable of selective gene regulation. Clin Exp Immunol. 1997;110(2):151-154. http://www.pubmedcentral. nih.gov/articlerender.fcgi?artid=2265499\&tool= pmcentrez\&rendertype=abstract. Accessed 15 Nov 2015.

10. Piura B, Medina L, Rabinovich A, Dyomin V, Huleihel M. Thalidomide distinctly affected TNF- $\alpha$, IL-6 and MMP secretion by an ovarian cancer cell line (SKOV-3) and primary ovarian cancer cells. Eur Cytokine Netw. 2013;24(3):122-9. doi:10.1684/ecn.2013.0342.

11. Song $\mathrm{T}$, Wang $\mathrm{L}, \mathrm{Gu} \mathrm{K}$, et al. Involvement of peripheral TRPV1 channels in the analgesic effects of thalidomide. Neurochem Int. 2015;85-86:40-5. doi:10.1016/j.neuint.2015.04.006.

12. Vargesson N. Thalidomide-induced teratogenesis: history and mechanisms. Birth Defects Res C Embryo Today. 2015;105(2):140-56. doi:10.1002/ bdrc. 21096 .

13. Muller GW, Chen R, Huang S-Y, et al. Amino-substituted thalidomide analogs: potent inhibitors of TNF- $\alpha$ production. Bioorg Med Chem Lett. 1999;9(11):1625-30. doi:10.1016/S0960894X(99)00250-4.

14. Kanavy H, Bahner J, Korman NJ. Treatment of refractory prurigo nodularis with lenalidomide. Arch Dermatol. 2012;148(7):794. doi:10.1001/ archdermatol.2011.2918.

15. Ferrandiz C, Carrascosa JM, Just M, Bielsa I, Ribera M. Sequential combined therapy with thalidomide and narrow-band (TL01) UVB in the treatment of prurigo nodularis. Dermatology. 1997;195:359-61.

16. van den Broek H. Treatment of prurigo nodularis with thalidomide. Arch Dermatol. 1980;116(5):571-2. doi:10.1001/archderm.1980. 01640290081018 .

17. Winkelmann RK, Connolly SM, Doyle JA, Padilha-Goncalves A. Thalidomide treatment of prurigo nodularis. Acta Derm Venereol. 1984;64(5):412-7.

18. Wulff $\mathrm{CH}$, Høyer $\mathrm{H}$, Asboe-Hansen G, Brodthagen H. Development of polyneuropathy during thalidomide therapy. $\mathrm{Br} \mathrm{J}$ Dermatol. 1985;112(4):475-80.

19. Crouch RB, Foley PA, Ng JCH, Baker CS. Thalidomide experience of a major Australian teaching hospital. Australas J Dermatol. 2002;43(4):278-84. doi:10.1046/j.1440-0960.2002. 00593.x.

20. Alfadley A, Al-Hawsawi K, Thestrup-Pedersen K, Al-Aboud K. Treatment of prurigo nodularis with 
thalidomide: a case report and review of the literature. Int $\mathrm{J}$ Dermatol. 2003;42(5):372-5. doi:10.1046/j.1365-4362.2003.01184.x.

21. Sharma NL, Sharma VC, Mahajan VK, Shanker V, Ranjan N, Gupta M. Thalidomide: an experience in therapeutic outcome and adverse reactions. J Dermatol Treat. 2007;18(0954-6634 (Print)): 335-340. doi:10.1080/09546630701386993.

22. Lan CCE, Lin CL, Wu CS, Chai CY, Chen WT, Chen GS. Treatment of idiopathic prurigo nodularis in Taiwanese patients with low-dose thalidomide. J Dermatol. 2007;34(4):237-42. doi:10.1111/j. 1346-8138.2007.00260.x.

23. Doherty SD, Hsu S. A case series of 48 patients treated with thalidomide. J Drugs Dermatol. 2008;7(8):769-73.

24. Orlando A, Renna S, Cottone M. Prurigo nodularis of hyde treated with low-dose thalidomide. Eur Rev Med Pharmacol Sci. 2009;13(2):141-5.
25. Andersen TP, Fogh K. Thalidomide in 42 patients with prurigo nodularis Hyde. Dermatology. 2011;223(2):107-12. doi:10.1159/000331577.

26. Taefehnorooz H, Truchetet F, Barbaud A, Schmutz JL, Bursztejn AC. Efficacy of thalidomide in the treatment of prurigo nodularis. Acta Derm Venereol. 2011;91(3):344-5. doi:10.2340/00015555-0997.

27. Liu H, Gaspari AA, Schleichert R. Use of lenalidomide in treating refractory prurigo nodularis. J Drugs Dermatol. 2013;12(3):360-361. http://www.ncbi.nlm.nih.gov/pubmed/23545923. Accessed 6 July 2015.

28. Rishi R, Ringwala S, Tracy J, Fatteh S. Prurigo nodularis and Hashimoto thyroiditis. Ann Allergy Asthma Immunol. 2014;113(6):673-4. doi:10.1016/ j.anai.2014.09.010.

29. Clemmensen OJ, Olsen PZ, Andersen KE. Thalidomide neurotoxicity. Arch Dermatol. 1984;120(3):338-41. 